\title{
TRANSPORT COEFFICIENTS IN HOT QCD
}

\author{
GUY D. MOORE \\ Department of Physics, McGill University, \\ 3600 rue University, Montreal, QC H3A 2T8, Canada
}

\begin{abstract}
I give a physical explanation of what shear viscosity is, and what physics determines its value. Then I explain why determining the shear viscosity of the Quark-Gluon Plasma is interesting. I outline the leading-order calculation of the QGP shear viscosity (and baryon number diffusion constant), explaining why the quite complicated physics of parton splitting and Landau-Pomeranchuk-Migdal interference effects are required for its calculation. Then I briefly explore the range of applicability, emphasizing the importance of plasma instabilities.
\end{abstract}

\section{Introduction}

In this talk I will discuss work with my collaborators, Peter Arnold and Larry Yaffe, on transport coefficients in QCD, particularly shear viscosity.

Interest in transport coefficients in the Quark-Gluon Plasma (QGP) started in the 1980's, and several rough estimates were made at that time ${ }^{1}$. The first really mature work was by Baym et al. ${ }^{2}$ in 1990. They showed that the dominant physics involved is small angle scattering, and they demonstrated how to perform a calculation accurate to leading order in the logarithm of the strong coupling, $\ln \left(1 / g_{\mathrm{s}}\right)$. We started work around 1999 under the mistaken impression that not much was required to improve their work to leading order in the coupling $g_{\mathrm{s}}$. After discovering that even the leading-log calculation was wrong in the literature, we decided to write two papers, rather than one-a quick paper ${ }^{3}$ computing the leading log correctly, and a longer one presenting a leading order calculation. The quick paper took 8 months and the "second" paper turned out to be six papers $4,5,6,7,8,9$, spanning the next two and a half years.

My goal is to explain how the physics of transport coefficients turns out to be much richer than we had anticipated. In Section 2, I will explain what shear viscosity is and what physics is involved; and I will briefly explain why it is an interesting property of the QGP. Section 3 will outline our calculation, emphasizing the roles played by soft scatterings, identity 
changing scatterings, and collinear processes. Then, Section 4 will briefly discusses how limited the range of the calculation is; not only does it depend on weak coupling, but on a near-equilibrium assumption outside of which the physics is completely different.

\section{The physics of shear viscosity}

The velocity of an ideal fluid evolve according to Euler's equations,

$$
\frac{\partial \boldsymbol{v}}{\partial t}=-\boldsymbol{v} \cdot \nabla \boldsymbol{v}-\frac{1}{\rho} \nabla p, \quad p=p(\rho) .
$$

Furthermore, the velocity at a boundary equals the velocity of that boundary (no slipping), and the stress tensor is determined by the pressure alone, that is, in the $\boldsymbol{v}=0$ frame it is given by

$$
T^{\mu \nu}=(\rho+p) u^{\mu} u^{\nu}+p \eta^{\mu \nu},
$$

(with $\eta^{\mu \nu}=\operatorname{Diag}[-,+,+,+]$ ). In fact, Euler's equations follow simply from the assumption that the stress tensor will satisfy this form, from an equation of state, and from stress-energy conservation, $\partial_{\mu} T^{\mu \nu}=0$.

Local equilibrium ensures that these conditions are met. What enforces local equilibrium is interactions between degrees of freedom; free theories never equilibrate. Therefore, the ideal description is a better and better approximation as a theory is more and more strongly coupled; in this case ideality is the opposite of free behavior.

To see this, consider a fluid undergoing shear flow. Shear flow means that, in the local rest frame, the traceless part of $\partial_{i} v_{j}$ is nonzero. The simplest example of shear flow is a fluid trapped between two plates, one fixed and one moving laterally with velocity $v$, as in Fig. 1; under ideal

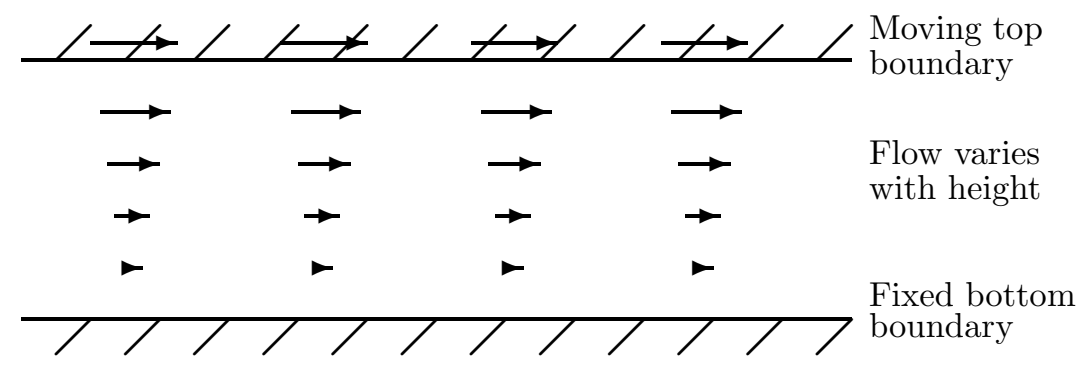

Figure 1. a system undergoing shear flow

Eulerian flow, the fluid at the top is moving with the upper boundary, with $T_{x z}=0$; therefore there is no lateral force on the top boundary. 
In a real fluid, there will be a "drag" force on the top plate; the shear viscosity is defined by the relation that the force per unit area is,

$$
\frac{F_{x}}{A}=T_{x z}=\eta \frac{d v_{x}}{d z}, \text { implying } T_{i j}=p \delta_{i j}+\eta\left(\partial_{i} v_{j}+\partial_{j} v_{i}-\frac{2}{3} \delta_{i j} \partial_{k} v_{k}\right) \text {. }
$$

Shear occurs because particles can fly freely. The particles near the top of the fluid region are primarily moving to the right; those near the bottom are moving right or left with equal likelihood. If the particles fly freely, the upward moving particles near the bottom, and the downward moving particles from the top, will meet in the middle, as shown in Fig. 2. However, the

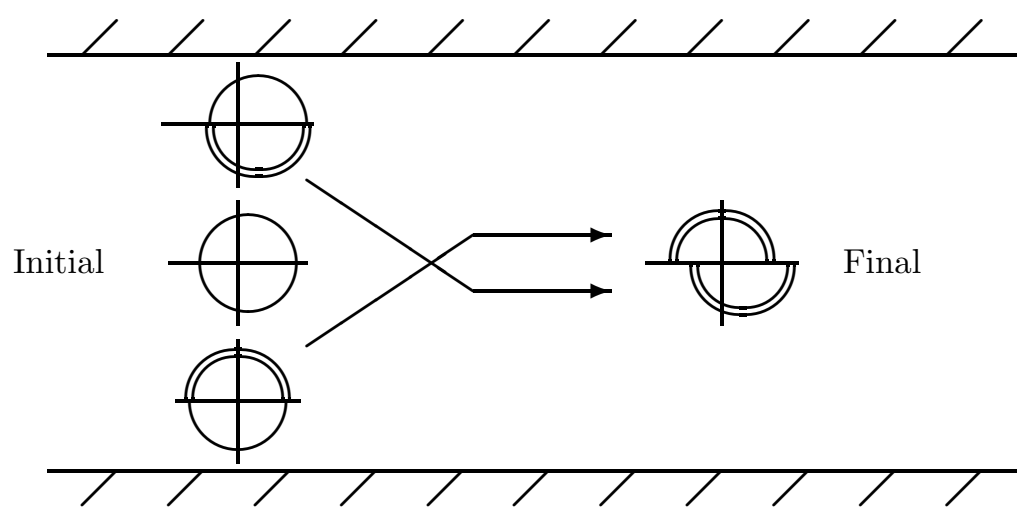

Figure 2. Free flow leads to an asymmetrical momentum distribution.

particles coming down from the top tend to be moving to the right, while particles coming up have no preferred direction; so the momentum distribution in the center (and everywhere, actually) tends to become skewed. Such a skewed distribution has nonvanishing $T_{x z}$ and there is net transverse momentum flow and a net drag on the top surface. Scatterings restore the distribution towards equilibrium, evening out the momentum distribution. The fewer scatterings, the more distorted the momentum distribution will be. Therefore, the shear viscosity will be smaller if the scattering rate is larger; $\eta \propto 1 / \Gamma$ with $\Gamma$ the mean scattering rate. ${ }^{\text {a }}$ The problem of deter-

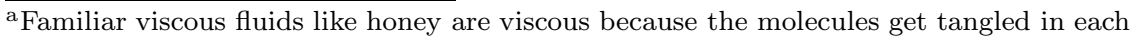
other, and are not a good analogy with any system which behaves approximately like a collection of freely flying objects. A better example is the air; as the pressure is lowered, there are fewer particles available to carry momentum, $T_{i j} \propto p$, so one would naively expect $\eta \propto p$. However, at lower pressure, collisions are rarer, $\Gamma \propto p$; and so the shear viscosity of air is actually independent of air pressure. 
mining the shear viscosity will be the problem of determining how collision processes re-arrange the momentum distribution of particles from skewed towards isotropic.

Why is the shear viscosity of the QGP interesting? One of the main focuses of this conference is the understanding of nonequilibrium dynamics; QCD is the most interesting theory to study this and viscosity and other transport coefficients (baryon number diffusion and electrical conductivity, which have very similar physics) are theoretically clean and well defined nonequilibrium dynamical quantities. That makes them ideal objects for "cutting our teeth" on calculational strategies for nonequilibrium field theories.

The shear viscosity of QCD is also directly interesting because it gives a handle on the quality of the hydrodynamical description of heavy ion collisions; this has been considered at some length recently by Teaney ${ }^{10}$, who found the corrections to flow parameters like $v_{2}$ due to viscosity at RHIC. Viscosity and particularly diffusion coefficients are also important in electroweak baryogenesis.

\section{The shear viscosity of the QGP}

Shear viscosity describes momentum transfer, and most of the momentum of a weakly coupled, near-equilibrium QGP is in quasiparticle excitations with momentum $p \sim 3 T$. These should be adequately described by kinetic theory, as I implicitly assumed in the last section's discussion. In fact, the validity of a kinetic description has essentially been derived diagrammatically, by Jeon for $\lambda \phi^{4}$ theory ${ }^{11}$ and by others for gauge theories ${ }^{12,13}$.

The physics of viscosity is the physics of direction changing scattering, and this is dominated by $2 \leftrightarrow 2 t$-channel gluon exchange ${ }^{1}$. In terms of the spatial momentum $q$ exchanged in the scattering process, such scatterings have a cross section of form,

$$
d \sigma \propto \frac{d^{3} q}{q} \frac{1}{q^{4}}
$$

which is quadratically small $q$ divergent - the familiar Coulomb divergence. However, what is important for shear viscosity is how quickly the particles change direction. Momentum changes from different scatterings add incoherently; so

$$
\int q^{2} d \sigma \propto \int q^{3} \frac{d^{3} q}{q} \frac{1}{q^{4}} \sim \int \frac{d q}{q}
$$


is the relevant quantity and enough collisions must occur for it to add up to $|\boldsymbol{p}|^{2}$, for a particle to change direction. This integral is log IR divergent. There are two key lessons.

(1) Small $q$ collisions dominate, but only by a logarithm.

(2) Large momentum particles take the longest time to change direction; since $(\delta \boldsymbol{p})^{2} \sim|\boldsymbol{p}|^{2}$ is required, the time scale for direction change goes as $\tau \propto|\boldsymbol{p}|^{2}$. Therefore high energy particles contribute disproportionally to $\eta$.

Baym et $a .^{2}{ }^{2}$ provided the first quantitative treatment which accounted for both of these points. To find the shear flow, they wrote the Boltzmann equation for the nonequilibrium distribution function $f(x, p)$,

$$
\frac{\partial f[p, x, t]}{\partial t}=-\boldsymbol{v} \cdot \nabla_{x} f[p, x, t]-\mathcal{C}[f][p, x, t],
$$

and linearized it. That is, they took $f=f_{0}+\delta f$ and expanded the collision integral $\mathcal{C}$ to first order in $\delta f$. To this order, one can write

$$
\delta f=f_{0}\left(1 \pm f_{0}\right) \partial_{i} v_{j}\left(\hat{p}_{i} \hat{p}_{j}-\frac{\delta_{i j}}{3}\right) \chi(p) .
$$

The collision integral is nonlocal in momentum space, so we have an integral equation to solve, which in general is nasty. They showed how to approach this variationally. They also showed that the physics of dynamical screening by the plasma, which is contained within the Hard Thermal Loops (HTL's) of Braaten and Pisarski ${ }^{14}$, is sufficient to render the Coulomb divergence finite; the would be logarithmic divergence becomes a large logarithm $\ln (1 / g)$. Further, at leading order in this logarithm $\mathcal{C}$ is semilocal in momentum, which allowed Baym at al. to solve for the shear viscosity of QCD at leading logarithmic order.

In our first paper ${ }^{3}$, we showed that, even at leading logarithmic order, one must also include Compton type processes,
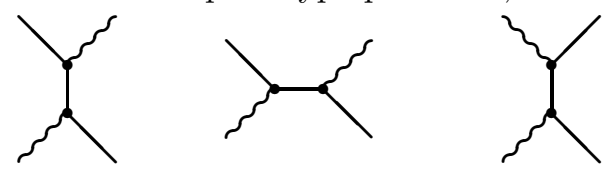

which are logarithmically IR divergent. The role of these processes is not to change particle direction, but to change particle identity; a quark on scattering becomes a gluon and vice versa. Because of their different group Casimir, gluons equilibrate more quickly than quarks; so the conversion of hard to equilibrate quarks into easy to equilibrate gluons accelerates thermalization. This is needed at leading-log order, though it is only numeri- 
cally important for electrical conductivity in QED. With these processes, a leading-log treatment is possible ${ }^{3}$, yielding, for instance,

$$
\begin{aligned}
\sigma & =\frac{12^{4} \zeta^{2}(3) \pi^{-3} N_{\text {lept }}}{3 \pi^{2}+32 N_{\text {spec }}} \frac{T}{e^{2} \ln 1 / e}, \\
D_{\text {quark }} & =\frac{2^{4} 3^{6} \zeta^{2}(3) \pi^{-3}}{24+4 N_{\mathrm{f}}+\pi^{2}} \frac{1}{g^{4} T \ln 1 / g} .
\end{aligned}
$$

The result for $\eta$ is more cumbersome and can be found in our paper ${ }^{3}$. The above results are only accurate to $0.5 \%$ because they were made with the Ansatz $\xi(p) \propto p^{2}$. More accurate results are presented in the paper.

What is required to convert these to a leading-order computation? One only needs to treat the collision integral more carefully, both in the $q \sim T$ momentum region where the collision integral is nonlocal, and in the $q \sim$ $m_{\mathrm{D}} \sim g_{\mathrm{s}} T$ region where screening effects must be dealt with. For heavy quark diffusion, this is sufficient; Derek Teaney and I recently derived a complete leading order result;

$$
D=\frac{72 \pi}{C_{\mathrm{f}} g_{\mathrm{s}}^{4} T}\left[\left(N_{\mathrm{c}}+\frac{N_{\mathrm{f}}}{2}\right)\left(\ln \frac{2 T}{m_{D}}+\frac{1}{2}-\gamma_{E}+\frac{\zeta^{\prime}(2)}{\zeta(2)}\right)+\frac{N_{\mathrm{f}} \ln 2}{2}\right]^{-1} .
$$

However, viscosity in the QGP and light quark diffusion turn out to involve more physics, and therefore more work; one must also include inelastic processes with collinear external states.

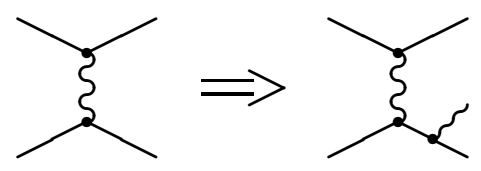

Particles in the plasma undergo the left process at an $O\left(\alpha_{\mathrm{s}} T\right)$ rate, not $\alpha_{\mathrm{s}}^{2} T$, because of the Coulomb divergence already discussed. The right process is slower by a factor of $\alpha_{\mathrm{s}}$; this is true even when the radiated gluon has momentum $O(T)$, provided it is collinear with the particle which emits it. The rate for this process is therefore as large as the large angle scattering rate.

We saw previously that the particles furthest from equilibrium were those with the most energy, $\delta f \propto f|\boldsymbol{p}|^{2}$. Collinear processes like the one just presented tend to split these high energy particles into lower energy ones, which are more easily bent; therefore they lead to equilibration and lower $\eta$, even though they do not change particle direction. Numerically, we find that they reduce $\eta$ by about $10 \%{ }^{9}$.

But life is even more complicated; one cannot ignore interference between emission amplitudes of form 


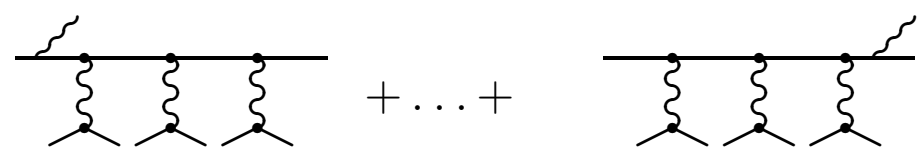

The effect of such interference is referred to as the Landau-PomeranchukMigdal (LPM) effect ${ }^{16}$, and it suppresses the efficiency of collinear emission. To see the physical origin of the suppression, consider a particle undergoing multiple scatterings, and remember that it possesses a finite transverse wave packet. That wave packet continues to physically overlap the wave packet of the emitted radiation for some time, called the formation time; if there is a second scattering in that time, the two radiation fields overlap and interfere;

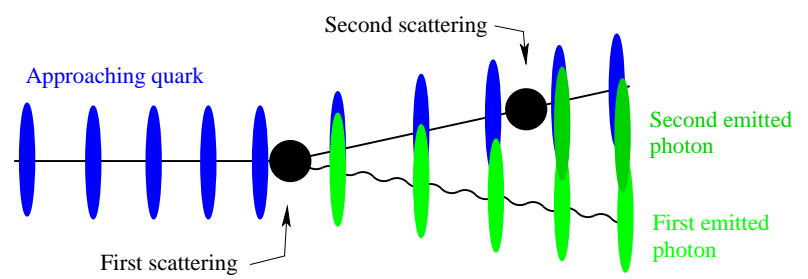

The radiations sum in amplitude; on average the interference is destructive.

Dealing with this complication takes a lot of work, and has been the topic of several studies ${ }^{17,5,7}$. The upshot is that the rate at which a particle of momentum $p$ undergoes a soft scattering which induces a bremsstrahlung of a gluon of energy $k$, is

$$
\begin{aligned}
\frac{d \Gamma(p, k)}{d k d t}= & \frac{C_{s} g_{\mathrm{s}}^{2}}{16 \pi p^{7}} \frac{1}{1 \pm e^{-k / T}} \frac{1}{1 \pm e^{-(p-k) / T}} \times\left\{\begin{array}{cc}
\frac{1+(1-x)^{2}}{x^{3}(1-x)^{2}} & q \rightarrow q g \\
N_{\mathrm{f}} \frac{x^{2}+(1-x)^{2}}{x^{2}(1-x)^{2}} & g \rightarrow q q \\
\frac{1+x^{4}+(1-x)^{4}}{x^{3}(1-x)^{3}} & g \rightarrow g g
\end{array}\right\} \times \\
& \times \int \frac{d^{2} \boldsymbol{h}}{(2 \pi)^{2}} 2 \boldsymbol{h} \cdot \operatorname{Re} \boldsymbol{F}(\boldsymbol{h}, p, k),
\end{aligned}
$$

where $\boldsymbol{F}$ is the solution of the following integral equation:

$$
\begin{aligned}
2 \boldsymbol{h}=i \delta E \boldsymbol{F}(\boldsymbol{h})+g^{2} \int \frac{d^{2} \boldsymbol{q}_{\perp}}{(2 \pi)^{2}} C\left(\boldsymbol{q}_{\perp}\right)\left\{\frac{2 C_{s}-C_{\mathrm{A}}}{2}\left[\boldsymbol{F}(\boldsymbol{h})-\boldsymbol{F}\left(\boldsymbol{h}-k \boldsymbol{q}_{\perp}\right)\right]+\right. \\
\left.+\frac{C_{\mathrm{A}}}{2}\left[\boldsymbol{F}(\boldsymbol{h})-\boldsymbol{F}\left(\boldsymbol{h}+p \boldsymbol{q}_{\perp}\right)+\boldsymbol{F}(\boldsymbol{h})-\boldsymbol{F}\left(\boldsymbol{h}-(p-k) \boldsymbol{q}_{\perp}\right)\right]\right\} \\
\delta E \equiv \frac{\boldsymbol{h}^{2}}{2 p k(p-k)}+\frac{m_{k}^{2}}{2 k}+\frac{m_{p-k}^{2}}{2(p-k)}-\frac{m_{p}^{2}}{2 p}
\end{aligned}
$$

with $C\left(\boldsymbol{q}_{\perp}\right)=\frac{m_{\mathrm{D}}^{2}}{\boldsymbol{q}_{\perp}^{2}\left(\boldsymbol{q}_{\perp}^{2}+m_{\mathrm{D}}^{2}\right)}, m_{\mathrm{D}}^{2}=\frac{g_{\mathrm{s}}^{2} T^{2}}{6}\left(2 N_{\mathrm{c}}+N_{\mathrm{f}}\right)$. Determining $d \Gamma / d k d t$ 
therefore requires the solution of an integral equation; and this for a collision term which, to solve the Boltzmann equation, must appear as a coefficient in an integral equation which is to be solved. However, the numerics turn out not to be very demanding.

These ingredients have been combined into a kinetic theory for the QGP 8 , which has been linearized and solved to determine the transport coefficients at leading order in the strong coupling ${ }^{9}$. Results for shear viscosity are presented in Fig. 3. At $\alpha_{\mathrm{s}}=1 / 3, \eta$ is quite small, and hydrodynamics should be nearly ideal; but the perturbative computation is breaking down at this point and the results cannot be trusted (factor of 3 uncertainty?).
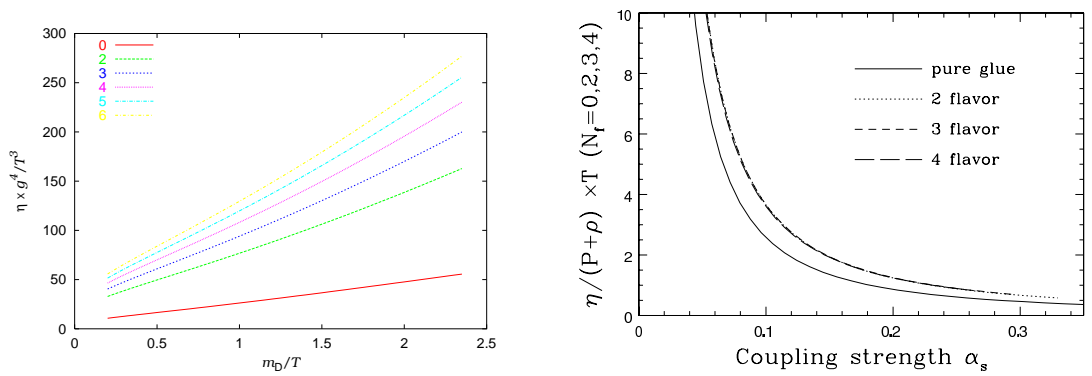

Figure 3. Shear viscosity, scaled by $\alpha_{\mathrm{s}}^{-2}$ (left) and by enthalpy density (right).

\section{Range of applicability of QGP transport coefficients}

Two key assumptions underlie the above calculation. The first is that the coupling is actually weak, at the scale relevant to the physics in question. For electroweak phase transition applications this is probably a good approximation; for RHIC it is dubious, though most of the theory community is guilty of this assumption. I will not discuss this more here.

The other assumption underpinning our treatment is, that the system is near equilibrium. One can check self-consistency by comparing the time scale of relaxation with the age of the plasma. The ratio $\eta /(\rho+p)$ is a time or length scale, called the sound attenuation length; it differs by a geometric factor $\sim 1 / 5$ from the relaxation time of the plasma. At $\alpha_{\mathrm{s}}=1 / 3$, Fig. 3 shows that this is $\sim 2 / 3 T$, so $\tau \sim 3 / T$, which is a few fermis at RHIC. Therefore we expect hydrodynamic treatments to be valid after $\sim 3$ fermi at RHIC. One might question a hydro description for times earlier than that. 
How different could the early time physics be? The answer turns out to be, pretty different. For systems which are far from equilibrium but approximately isotropic, kinetic theory should be applicable under fairly weak conditions $^{8}$. But for systems with strong momentum space anisotropy, this turns out not to be the case. A treatment involving soft gauge fields becomes essential, because these soft gauge fields show exponential growth due to plasma instabilities ${ }^{18}$. It has recently been noted that this may be important for QGP physics 19,20,21, and it is the topic of two other talks at this conference ${ }^{22,23}$. The short story is that, for plasmas which are far from isotropic, soft gauge field modes can grow at a rate suppressed by only a single power of $g_{\mathrm{s}}$, and may ${ }^{24}$ randomize the directions of other particles on this time scale.

\section{Conclusions}

Transport coefficients in gauge theories can be computed within kinetic theory, but they involve rich physics; hard thermal loops, multiple scattering processes, collinear splitting. The kinetic description is only possible after resumming certain multiple scattering emission processes to account for LPM interference. It has taken nearly 20 years to go from the first attempts at a quantitative analysis to one which is complete at leading order in the coupling $g_{\mathrm{s}}$.

The calculation of transport coefficients has been directly useful to the heavy ion community ${ }^{10}$, but it has also been a good object lesson in nonequilibrium field theory in QCD. Most importantly, we have learned just how narrow the domain of validity of kinetic theory is, and how much more complicated the physics of highly anisotropic plasmas may be. The problem of understanding plasma instabilities in QCD is only beginning to be addressed and should be the subject of much future work.

\section{Acknowledgements}

I particularly thank my co-workers, Peter Arnold and Larry Yaffe. I also thank Mike Strickland, Stan Mrówczyński, and Francois Gelis for conversations which have been useful in this work over the past years. My work is currently supported by grants from the Natural Sciences and Engineering Research Council of Canada and by le Fonds Québécois de la Recherche sur la Nature et les Technologies. 


\section{References}

1. A. Hosoya, M. Sakagami and M. Takao, Annals Phys. 154, 229 (1984); A. Hosoya and K. Kajantie, Nucl. Phys. B250, 666 (1985).

2. G. Baym, H. Monien, C. J. Pethick and D. G. Ravenhall, Phys. Rev. Lett. 64, 1867 (1990); Nucl. Phys. A525, 415C (1991).

3. P. Arnold, G. D. Moore and L. G. Yaffe, JHEP 0011, 001 (2000) [hep$\mathrm{ph} / 0010177]$.

4. G. D. Moore, JHEP 0105, 039 (2001) [hep-ph/0104121].

5. P. Arnold, G. D. Moore and L. G. Yaffe, JHEP 0111, 057 (2001) [hepph/0109064].

6. P. Arnold, G. D. Moore and L. G. Yaffe, JHEP 0112, 009 (2001) [hep$\mathrm{ph} / 0111107]$.

7. P. Arnold, G. D. Moore and L. G. Yaffe, JHEP 0206, 030 (2002) [hep$\mathrm{ph} / 0204343]$.

8. P. Arnold, G. D. Moore and L. G. Yaffe, JHEP 0301, 030 (2003) [hepph/0209353].

9. P. Arnold, G. D. Moore and L. G. Yaffe, JHEP 0305, 051 (2003) [hep$\mathrm{ph} / 0302165]$.

10. D. Teaney, Phys. Rev. C 68, 034913 (2003) [nucl-th/0301099].

11. S. Jeon, Phys. Rev. D 52, 3591 (1995) [hep-ph/9409250]; S. Jeon and L. G. Yaffe, Phys. Rev. D 53, 5799 (1996) [hep-ph/9512263].

12. G. Aarts and J. M. Martinez Resco, JHEP 0211, 022 (2002) [hepph/0209048]; G. Aarts and J. M. Martinez Resco, Phys. Rev. D 68, 085009 (2003) [hep-ph/0303216].

13. M. A. Valle Basagoiti, Phys. Rev. D 66, 045005 (2002) [hep-ph/0204334].

14. E. Braaten and R. D. Pisarski, Nucl. Phys. B 337, 569 (1990).

15. D. Teaney and G. D. Moore, in preparation.

16. L. D. Landau and I. Pomeranchuk, Dokl. Akad. Nauk Ser. Fiz. 92 (1953) 535; Dokl. Akad. Nauk Ser. Fiz. 92 (1953) 735; A. B. Migdal, Dokl. Akad. Nauk S.S.S.R. 105, 77 (1955); Phys. Rev. 103, 1811 (1956).

17. See for instance, R. Blankenbecler and S. D. Drell, Phys. Rev. D 53, 6265 (1996); R. Baier, Y. L. Dokshitzer, A. H. Mueller, S. Peigne and D. Schiff, Nucl. Phys. B 478, 577 (1996) [hep-ph/9604327]; B. G. Zakharov, Phys. Atom. Nucl. 61, 838 (1998) [Yad. Fiz. 61, 924 (1998)] [hep-ph/9807540].

18. E. S. Weibel, Phys. Rev. Lett. 2, 83 (1959).

19. S. Mrówczyński, Phys. Lett. B 214, 587 (1988); Phys. Lett. B 314, 118 (1993); Phys. Lett. B 393, 26 (1997) [hep-ph/9606442].

20. P. Romatschke and M. Strickland, Phys. Rev. D 68, 036004 (2003) [hepph/0304092]; hep-ph/0406188.

21. P. Arnold, J. Lenaghan and G. D. Moore, JHEP 0308, 002 (2003) [hepph/0307325].

22. P. Romatschke and M. Strickland, these proceedings, hep-ph/0408314.

23. Peter Arnold, these proceedings.

24. P. Arnold and J. Lenaghan, hep-ph/0408052. 